Special report

\title{
Working meeting on blood pressure measurement: suggestions for measuring blood pressure to use in populations surveys
}

\author{
Pan American Hypertension Initiative ${ }^{1}$
}

ABSTRACT As part of the Pan American Hypertension Initiative (PAHI), the Pan American Health Organization and the National Heart, Lung, and Blood Institute of the National Institutes of Health of the United States of America conducted a working meeting to discuss blood pressure $(B P)$ measurement methods used in various hypertension prevalence surveys and clinical trials, with the objective of developing a BP measurement protocol for use in hypertension prevalence surveys in the Americas. No such common protocol has existed in the Americas, so it has been difficult to compare hypertension prevention and intervention strategies. This piece describes a proposed standard method for measuring blood pressure for use in population surveys in the Region of the Americas. The piece covers: considerations for developing a common blood pressure measurement protocol, critical issues in measuring blood pressure in national surveys, minimum procedures for blood pressure measurement during surveillance, and quality assessment of blood pressure.

Key words Blood pressure, blood pressure determination, hypertension, guidelines, population surveillance.

On 12-13 June 2000 the National Heart, Lung, and Blood Institute and the Pan American Health Organization conducted a working meeting to discuss blood pressure (BP) measurement methods used in various hypertension prevalence surveys and clinical trials. This workshop is part of the Pan American Hypertension Initiative

\footnotetext{
Pan American Hypertension Initiative, Executive Secretariat, c/o Edward J. Roccella, National High Blood Pressure Education Program, National Heart, Lung, and Blood Institute, 31 Center Drive MSC 2480, Bethesda, Maryland 20892-2480, United States of America; e-mail: roccella@nih.gov
}

(PAHI) (1). The objective of the working meeting was to develop a BP measurement protocol for use in hypertension prevalence surveys in the Americas. No such common protocol existed in the Americas, thus it has been difficult to compare hypertension prevention and intervention strategies when different procedures are used to measure BP. For example, some BP prevalence studies define hypertension as 160/95 mm $\mathrm{Hg}$ while others use 140/90 mm Hg. While studies define the diastolic pressure as the fifth Korotkoff (K5) sound heard, some studies state the criteria as the disap- pearance of sound, and others as the onset of silence, which is $2 \mathrm{~mm} \mathrm{Hg}$ below $\mathrm{K} 5$. In addition, studies vary regarding the numbers of BP measurements taken, equipment used, training methods, and verification procedures.

\section{CONSIDERATIONS FOR DEVELOPING A COMMON BLOOD PRESSURE MEASUREMENT PROTOCOL}

Differences in BP definition and measurement criteria have the potential to produce substantial differences 
in hypertension prevalence. Thus data cannot be compared or pooled, and the advantages of examining populations with large sample sizes are lost. Further, changes or improvements in hypertension control rates or average BPs cannot be measured over time and therefore community intervention programs and activities cannot be evaluated. In addition, it is important to educate technicians in the proper calibration of equipment, the use of proper BP cuff size, and the avoidance of measurement digit preference.

\section{CRITICAL ISSUES IN MEASURING BLOOD PRESSURE IN NATIONAL SURVEYS}

Because of short-term variability in BP within a person, multiple measures should be taken on at least one occasion. Three BPs should be taken at one sitting, preferably prior to intensive interview or examination procedures, such as blood drawing, that may also be part of the survey.

Either a standard mercury sphygmomanometer or a certified automated BP device should be used. Mercury sphygmomanometers have been the predominant BP measurement device, though they are being phased out of service in Europe and the United States of America due to environmental concerns about mercury spillage from broken equipment and cleanup costs, which are extensive (2). Automated devices may take on an increased role in BP surveillance studies and are now being used. However, automated devices must be calibrated against a mercury standard or validated digital pressure meter, and they should pass equipment standards developed by the British Hypertension Society (3) or the Association for the Advancement of Medical Instrumentation (4). Devices designed to measure BP from the finger or the wrist are inappropriate for surveillance work. Aneroid sphygmomanometers are less reliable, but if used at all, they require frequent calibration (5)
Technicians must be trained, initially certified, and periodically recertified in $\mathrm{BP}$ measurement procedures and equipment care. Appropriate cuff sizes-as many as four different sizes-should be made available. If a stethoscope is necessary, it should include a bell. A brief questionnaire is required to determine whether the participants were previously diagnosed for hypertension and whether they were taking antihypertensive medications at the time of the survey. To assure continual quality measurements, technicians need to be evaluated regularly by supervisors, and quality of performance needs to be periodically assessed using statistical tables to detect bias in technicians' measurements. Technician retraining may be necessary to maintain the acquired skills.

\section{MINIMUM PROCEDURES FOR BLOOD PRESSURE MEASUREMENT DURING SURVEILLANCE}

The procedures to accurately measure BP are:

1. Participant sits at a table quietly with both feet flat on the floor and with the back supported. The bladder should be empty. The room should be comfortable and noise minimized. No tobacco products, alcohol, or caffeine should have been consumed within the previous 30 minutes. If this is not possible, it should be noted in the data.

2. The right arm, which should be bare, is placed on the table (at heart level) slightly flexed, with the palm upward. The screener should be in position to see the manometer at eye level.

3. Determine arm circumference and select and wrap appropriate cuff size. The lower edge of the cuff must be $2.5 \mathrm{~cm}$ above the elbow joint.

4. Wait 5 minutes.

5. Palpate radial pulse and inflate to $30 \mathrm{~mm} \mathrm{Hg}$ above the level where radial pulse disappears (peak inflation level). Deflate cuff.

6. Wait 30 seconds before re-inflating.

7. Inflate to peak inflation level.

8. Deflate at $2 \mathrm{~mm} \mathrm{Hg}$ per second.

9. Record the systolic BP, Korotkoff phase 1 (first of at least two regular consecutive sounds). Record to the nearest even number.

10. Record diastolic BP, Korotkoff phase 5 (the end of the last sound heard). Record to the nearest even number.

11. Finish deflation, then raise participant's arm above heart level for 15 seconds. Rest for one minute and then perform the measurement procedures two more times. Use the mean of the last two measurements.

When using automated devices, points 1, 2, 3, 4, and 11 are required. Point 5 depends on whether the automated device senses pressure during inflation. The other points are not applicable to automated devices. All points are applicable when using manual auscultatory devices.

\section{QUALITY ASSESSMENT OF BLOOD PRESSURE}

The following issues should be considered prior to survey development. Digit preference issues are usually applicable to manual devices but have also been demonstrated in automated devices.

Quality control analyses can identify technicians and/or procedures that need correction. Since deflation of the cuff is at $2 \mathrm{~mm}$ HG per second and readings are made to the nearest even number, a check should be made on the uniformity of all even-numbered digits. This is done by counting the observed number of measurements ending in each of the even digits and calculating a chi-square statistic and a digit preference score. Since at least two BP measurements are made, technicians with extreme differences in these two measurements, or more than expected identical measurements, should receive 
additional training. Technicians may not be able to complete all BPs (identified by a table of incomplete data), but if this is more frequent in some, then further training is required. Mean val- ues of BPs at sequential calendar time periods should be calculated to determine whether there is a shift (sudden change) or drift (gradual change) in the mean values over time. This as- sumes that participants are similar to each other over the time of the study. These quality control analyses are described in detail in a report by the World Health Organization (6).

\title{
REFERENCES
}

1. Pan American Hypertension Initiative. Pan American Hypertension Initiative [Internet site]. Available from: http://www.mco.edu/ org/whl/pahi.html. Accessed 12 September 2003.

2. United States of America, National Institutes of Health. Mad as a hatter? Join our campaign for a mercury-free NIH [Internet page]. Available from: http://www.nih.gov/od/ors/ds/ nomercury/index.htm. Accessed 12 September 2003.

3. British Hypertension Society. Blood pressure monitors [Internet page]. Available from: http://www.hyp.ac.uk/bhsinfo/bpmindex. html. Accessed 12 September 2003.

4. Association for the Advancement of Medical Instrumentation. Association for the Advancement of Medical Instrumentation [Web site]. Available from: http://www.aami.org. Accessed 12 September 2003

5. Canzanello VJ, Jensen PL, Schwartz GL Are aneroid sphygmomanometers accurate in hospital and clinic settings? Arch Intern Med 2001; 161(5):729-731.

6. Kuulasmaa K, Hense H-W, Tolonen H. Quality assessment of data on blood pressure in the
WHO MONICA Project. Geneva: World Health Organization and the WHO MONICA Project; 1999. Available from: http://www.ktl. fi/publications/monica/bp/bpqa.htm [Internet page]. Accessed 12 September 2003.

Manuscript received 14 August 2003. Revised version accepted for publication on 25 September 2003.

\section{International Conference on Women and Infectious Diseases}

\author{
Dates: $\quad$ 27-28 February 2004 \\ Location: Marriott Marquis Hotel \\ Atlanta, Georgia, United States of America
}

In collaboration with various other partners, the National Center for Infectious Diseases of the Centers for Disease Control and Prevention of the United States of America is organizing the International Conference on Women and Infectious Diseases (ICWID). The goal of the Conference is to enhance prevention and control of infectious diseases among women worldwide. The ICWID will consist of several plenary sessions as well as breakout sessions. Featured topics will include the impact of globalization, women and HIV/AIDS, perinatal infectious diseases, vaccinations, and links between infectious and chronic diseases. Other topics will include infectious disease disparities, sex-appropriate interventions, effective health communications, and cultural competence in preventing infectious diseases among women. The ICWID event will be held on the two days preceding the International Conference on Emerging Infectious Diseases, at the same hotel. The registration fee for the ICWID meeting is US\$ 175 if paid by 14 February 2004, and it is US\$ 225 after that date.

\author{
Information: \\ Marian McDonald \\ $\mathrm{NCID} / \mathrm{CDC}$ \\ Telephone: 404-371-5312 \\ E-mail: mmcdonald@cdc.gov \\ Internet: http://www.womenshealthconf.org.
}

